\title{
Novel Ti3C2Tx MXene Nanozyme with Manageable Catalytic Activity and Application to Electrochemical Biosensor
}

\section{Rongjun Yu}

University-Town Hospital of Chongqing Medical University

Jian Xue

Chongqing Medical University

\section{Yang Wang}

University-Town Hospital of Chongqing Medical University

Jingfu Qiu

Chongqing Medical University

\section{Xinyi Huang}

Chongqing Medical University

\section{Anyi Chen}

Chongqing Medical University

jianjiang xue ( $\nabla$ jianjiangxue@163.com )

University-Town Hospital of Chongqing Medical University

\section{Research Article}

Keywords: Ti3C2Tx MXene, Nanozyme, Electrocatalysis, Cascading catalytic amplification, Electrochemical biosensor

Posted Date: October 19th, 2021

DOl: https://doi.org/10.21203/rs.3.rs-965061/v1

License: (a) (1) This work is licensed under a Creative Commons Attribution 4.0 International License. Read Full License 


\section{Abstract}

In this work, $\mathrm{Ti}_{3} \mathrm{C}_{2} \mathrm{~T}_{\mathrm{x}} \mathrm{MXene}$ was identified as efficient nanozyme with area-dependent electrocatalytic activity in oxidation of phenolic compounds, which originated from the strong adsorption effect between the phenolic hydroxyl group and the oxygen atom on the surface of $\mathrm{Ti}_{3} \mathrm{C}_{2} \mathrm{~T}_{\mathrm{x}} \mathrm{MXene}$ flake. On the basis of the novel electrocatalytic activity, $\mathrm{Ti}_{3} \mathrm{C}_{2} \mathrm{~T}_{\mathrm{x}} \mathrm{MXene}$ was combined with alkaline phosphatase to construct a novel cascading catalytic amplification strategy using 1-naphthyl phosphate (1-NPP) as substrate, thereby realizing efficient electrochemical signal amplification. Taking advantage of the novel cascading catalytic amplification strategy, an electrochemical biosensor was fabricated for BCR/ABL fusion gene detection, which achieved excellent sensitivity with linear range from $0.2 \mathrm{fM}$ to $20 \mathrm{nM}$ and limit of detection down to $0.05 \mathrm{fM}$. This biosensor provided a promising tool for ultrasensitive fusion gene detection in early diagnosis of chronic myelogenous leukemia and acute lymphocytic leukemia. Moreover, the manageable catalytic activity of MXene broke a path for developing nanozymes, which possessed enormous application potential in not only electrochemical analysis but also the extensive fields including organic synthesis, pollutant disposal and so on.

\section{Introduction}

Electrochemical biosensor has become one of the most predominant analysis tools in clinical diagnosis due to the outstanding merits of high sensitivity and selectivity, rapid response, low cost, simple instrumentation, easy miniaturization and good quantitative ability[1-4]. Enzymatic electrocatalysis is a widely used technique in electrochemical biosensor, which improves the analytical sensitivity by promoting the electrochemical redox reactions with the help of enzymes[5-7]. Nevertheless, natural enzymes are general cost to manufacture and store, unstable to transfer or modify, and sensitive to harsh physiochemical conditions. Nanozymes, nanomaterials with enzyme-like catalytic activities, well overcome the shortages of natural enzymes[8]. Therefore, nanozymes are attracting increasing attention in bioanalysis to substitute conventional natural enzyme[9]. However, the catalytic activities of conventional nanozymes strongly rely on the defect dependent active centers, such as surface dangling bonds or heterogeneous doping domains, which is distributed unevenly on the surface[10,11]. As a consequence, the homogeneity of particle is a very important parameter of nanozymes, which brings great challenge to prepare high-quality product and applied to precise quantitative analysis[9]. From this prospective, developing nanomaterials with enzyme-like catalytic activities independent to the morphology or crystal defect is of great significance as this kind of materials might be more easily controlled to obtain uniform catalysis activity.

MXene is termed for a series of two-dimension (2D) transition metal carbides, nitrides, and carbonitrides[12, 13]. Recent years, the electrocatalytic activity MXene have attracted enormous research interest in diverse fields, such as hydrogen evolution[14], oxygen evolution[15, 16], $\mathrm{N}_{2}$-to- $\mathrm{NH}_{3}$ conversion[17], fuel cell[18, 19] and carbon dioxide reduction[20, 21]. These reported works implied a probable reality that the catalysis activities of MXene could be originated from the $2 \mathrm{D}$ basal planes rather 
than the defects, which was significantly different from conventional nanozymes including metallic oxide[22-24], carbon nanomaterials[25, 26], and 2D metallic sulfide[27, 28]. As a result, the electocatalytic activity of MXene was directly related to the area of flake rather than the shape or morphology. Benefiting from the unique electrocatalytic characteristics and 2D structure, MXenes were expected to provide a chance to easily control the electrocatalytic activity by simply customize the total area of flakes.

In this work, the electrocatalytic activity of $\mathrm{Ti}_{3} \mathrm{C}_{2} \mathrm{~T}_{\mathrm{x}}$ MXene for phenols oxidation was identified and applied to constructing a cascading catalytic amplification strategy for electrochemical biosensor to determine of $B C R / A B L$ fusion gene, the key biomarker for clinical diagnosis of chronic myeloid leukemia (CML). $\mathrm{Ti}_{3} \mathrm{C}_{2} \mathrm{~T}_{x} \mathrm{MXene}$ presented efficient and area-dependent phenol adsorption on the $2 \mathrm{D}$ plane, thus catalyzing the electrochemical oxidation. For biosensor application, $\mathrm{Ti}_{3} \mathrm{C}_{2} \mathrm{~T}_{\mathrm{x}} \mathrm{MXene}$ was spread on electrode and further decorated with gold nanoparticles for DNA capture probe (CP) immobilization. Besides, DNA walking machine was employed to recognize target $B C R / A B L$ fusion gene and mediate nucleic acid amplification. As illustrated in Scheme 1, the DNA walking machine would start DNA nicking and expose DNA fragments from the magnetic beads in the presence of BCR/ABL fusion gene. The exposed DNA fragments helped the assembly of biotin labeled DNA probe (Bio-DP) on the sensing surface according to the sandwich DNA hybridization of CP-DNA fragment-Bio-DP. Finally, streptavidin modified alkaline phosphatase (SA-ALP) further was modifed onto the biosensing interface via the specific biotin-streptavidin reaction. With the addition of 1-naphthyl phosphate (1-NPP) in the electrolyte solution, 1-naphthol was produced via ALP-catalytic hydrolysis of 1-NPP and generated an amplified electrochemical signal via $\mathrm{Ti}_{3} \mathrm{C}_{2} \mathrm{~T}_{\mathrm{x}}$ MXene-catalytic electrochemical oxidization. With DNA walking machine and cascading catalysis for signal amplification, the electrochemical biosensor achieved excellent sensitivity for detection of BCR/ABL fusion gene with the linear range from $0.2 \mathrm{fM}$ to $20 \mathrm{nM}$ and limit of detection down to $0.05 \mathrm{fM}$, which could provide a powerful bioanalysis tool for clinical diagnose of CML. Moreover, the efficient electrocatalysis activity of $\mathrm{Ti}_{3} \mathrm{C}_{2} \mathrm{~T}_{\mathrm{x}} \mathrm{MXene}$ for phenols oxidation possessed great application potential in the more fields including sewage treatment and organic synthesis and so on.

\section{Experimental Section}

\subsection{Reagents and materials}

$\mathrm{Ti}_{3} \mathrm{C}_{2} \mathrm{~T}_{\mathrm{x}}$ (MXene) few layer dispersion solution (Lateral size 2-5 $\mu \mathrm{m}$ ), $\mathrm{Fe}_{3} \mathrm{O}_{4}$ nanoparticles, $\mathrm{TiO}_{2}$ nanoparticles, bulk $\mathrm{Ti}_{3} \mathrm{AlC}_{2}$ and $\mathrm{WS}_{2}$ nanosheets (Diameter 2-5 $\mu \mathrm{m}$ ) were obtained from Jiangsu XFNANO Materials Tech. Co., Ltd. (Nanjing, China). $\mathrm{MoS}_{2}$ nanosheets (Diameter 20-500 nm) were obtained from Nanjing JCNANO Tech. Co., Ltd. (Nanjing, China). $\mathrm{NH}_{2}-\mathrm{Fe}_{3} \mathrm{O}_{4}$ and Nafion solutions were obtained from Aladdin Biochemical Tech. Co., Ltd. (Shanghai, China). Gold chloride $\left(\mathrm{HAuCl}_{4} \bullet 4 \mathrm{H}_{2} \mathrm{O}\right)$, sodium citrate, 6mercaptohexanol (MCH), 1-naphthyl phosphate (1-NPP), 1-naphthol, streptavidin-alkaline phosphatase (SA-ALP), 4-nitrophenol, $\beta$-estradiol and diethanolamine (DEA) were purchased from Sigma-Aldrich 
Chemical (St. Louis, USA). Tris(2-carboxyethyl) phosphine hydrochloride (TCEP) was purchased from Sangon Biotech. Co., Ltd. (Chongqing, China). Nt.BsmAl nicking endonuclease (Nt.BsmAl) and CutSmart buffer were provided by New England Biotech. Co., Ltd. (Beijing, China). All high-performance liquid chromatography (HPLC)-purified sequences (Table S1) in our experiments were ordered from Sangon Biotech. Co., Ltd. (Shanghai, China). Clinical serum samples were obtained from the University-Town Hospital of Chongqing Medical University (Chongqing, China). The buffers and solutions involved in this experiment were display in Supplementary Material S1.

\subsection{Modification of electrode surface}

Prior to modification, the bare glassy carbon electrode (GCE) was polished with $300 \mathrm{~nm}$ and $50 \mathrm{~nm}$ alumina slurries to a mirror-like surface and then rinsed ultrasonically with ultrapure water, anhydrous ethanol and ultrapure water for $5 \mathrm{~min}$ in sequence. Then, the GCE was dried by nitrogen at room temperature. Simultaneously, $\mathrm{Ti}_{3} \mathrm{C}_{2} \mathrm{~T}_{x}$ MXene $(0.1 \mathrm{mg} / \mathrm{mL})$ was suspended in ultrapure water containing a $0.1 \%$ Nafion solution and sonicated for $60 \mathrm{~min}$. Gold nanoparticles (AuNPs) were synthesized according to a typical method, and the detailed procedure was elaborated in Supplementary Material S3. The products were stored at $4^{\circ} \mathrm{C}$ protected from light for further use.

Next, $10 \mu \mathrm{L}$ of the $\mathrm{Ti}_{3} \mathrm{C}_{2} \mathrm{~T}_{x}$ MXene $(0.10 \mathrm{mg} / \mathrm{mL})$ suspension was dropped onto the GCE surface and allowed to dry slowly, followed by the addition of $10 \mu \mathrm{L}$ AuNPs solution and drying at room temperature to obtain the modified electrodes (AuNPs/ $\mathrm{Ti}_{3} \mathrm{C}_{2} \mathrm{~T}_{\mathrm{x}} \mathrm{MXene/GCE}$ ). Afterwards, $10 \mu \mathrm{L}$ of thiolated capture probe (CP) pretreated by TCEP was dropped onto the AuNPs/ $\mathrm{Ti}_{3} \mathrm{C}_{2} \mathrm{~T}_{\mathrm{x}} \mathrm{MXene/GCE}$ surface and incubated overnight at $4^{\circ} \mathrm{C}$. After being washed with washing buffer, the modified electrode was further incubated with $1.0 \mathrm{mM} \mathrm{MCH}$ for $1 \mathrm{~h}$ at room temperature to block the nonspecific site, obtaining the electrochemical biosensing platform ( $\mathrm{MCH} / \mathrm{CP} / \mathrm{AuNPs} / \mathrm{Ti}_{3} \mathrm{C}_{2} \mathrm{~T}_{\mathrm{x}} \mathrm{MXene/GCE}$ ).

\subsection{Preparation of DNA walking machine}

DNA walking machine was prepared with reference to a previous report[29]. First, $1.0 \mu \mathrm{L}$ walker probe solution $(2.0 \mu \mathrm{M})$ and $1.0 \mu \mathrm{L}$ protecting probe solution $(2.0 \mu \mathrm{M})$ were mixed and heated at $95^{\circ} \mathrm{C}$ for 5 min, and then naturally cooled to obtain ds-DNAs. Later, $20 \mu \mathrm{L}$ support probe solution $(2.0 \mu \mathrm{M})$ was sufficiently mixed with dsDNA and added to $20 \mu \mathrm{L} \mathrm{Au@Fe} \mathrm{O}_{3}$ (the detailed procedure was described in Supplementary Material S4), stirred overnight and magnetically separated to obtain the expected DNA walking machine (DNA-Au@Fe $\mathrm{O}_{4}$ ). Finally, the well prepared DNA walking machine was further suspended in PBS and stored at $4^{\circ} \mathrm{C}$ for further use.

\subsection{Procedure for $B C R / A B L$ fusion gene detection}

First, BCR/ABL fusion gene standard sample $(1.0 \mu \mathrm{L})$ at different concentrations, $10 \mathrm{U} \mathrm{Nt.BsmA} \mathrm{X}$ and were added in to the dispersion of DNA walking machine and kept for $2 \mathrm{~h}$ at $37^{\circ} \mathrm{C}$. Then, supernatant solution was collected numerous after magnetic separation, which contained the produced intermediate DNAs. After that, $10 \mu \mathrm{L}$ of the above supernatant solution and $10 \mu \mathrm{L}$ of $2.5 \mu \mathrm{M}$ biotinylated detection probe were dipped onto the electrode and incubated at $37^{\circ} \mathrm{C}$ for $1 \mathrm{~h}$. Rinsing with washing buffer, the 
obtained electrode was treated in $10 \mu \mathrm{L}$ of DEA buffer containing $1.25 \mu \mathrm{g} / \mathrm{mL}$ ST-ALP and $8 \mathrm{mg} / \mathrm{mL} \mathrm{BSA}$ at $37^{\circ} \mathrm{C}$ for $30 \mathrm{~min}$. Finally, the electrochemical signal was measured in the DEA buffer containing 1.0 $\mathrm{mg} / \mathrm{mL}$ 1-NPP by differential pulse voltammetry (DPV) after rinsing with DEA buffer to remove the unbound SA-ALP. All parameter configurations of electrochemical measurements were shown in Supplementary Material S5.

\subsection{Theoretic calculation methods}

The first principle calculations are performed by Vienna Ab initio Simulation Package (VASP)[30] with the projector augmented wave (PAW) method[31]. The exchange-functional is treated using the PerdewBurke-Ernzerhof (PBE)[32] functional, in combination with the DFT-D correction[33]. The cut-off energy of the plane-wave basis is set at $500 \mathrm{eV}$. For the optimization of both geometry and lattice size, the Brillouin zone integration is performed with $2 * 2 * 1$ Monkhorts-Pack k-point sampling. The self-consistent calculations apply a convergence energy threshold of $10^{-5} \mathrm{eV}$. The equilibrium geometries and lattice constances are optimized with maximum stress on each atom within $0.02 \mathrm{eV} / \AA$.

\section{Results And Discussion}

\subsection{Morphological and elemental analysis of the $\mathrm{Ti}_{3} \mathrm{C}_{2} \mathrm{~T}_{\mathrm{x}}$ MXene}

Transmission electron microscopy (TEM) was employed to study the morphology of the used $\mathrm{Ti}_{3} \mathrm{C}_{2} \mathrm{~T}_{\mathrm{x}}$ MXene sample, which presented a remarkably large flake and some stacked little fragments (Fig. 1A). Moreover, the high-angle annular dark-field (HAADF)-STEM image showed no observable spot on the flake, suggesting the uniform distribution of the elements (Fig. 1B). As shown in Fig. 1C-1E, STEM-EDS elemental mappings of $\mathrm{C}, \mathrm{Ti}$, and $\mathrm{O}$ presented outlines well matched with the HAADF-STEM image, which visually displayed the elemental composition of the $\mathrm{Ti}_{3} \mathrm{C}_{2} \mathrm{~T}_{\mathrm{x}} \mathrm{MXene}$.

Energy dispersive X-ray spectroscopy (EDX) was also utilized to analyze the elemental composition of the $\mathrm{Ti}_{3} \mathrm{C}_{2} \mathrm{~T}_{\mathrm{x}}$ MXene (see in Supplementary Material, Fig. S1), which involved of C, O, Ti, F and Al elements. Among them, $\mathrm{F}$ and $\mathrm{Al}$ were mainly from the residual impurities and their contents were notably lower than $\mathrm{C}, \mathrm{O}$, and $\mathrm{Ti}$.

Atomic force microscope (AFM) was employed to further study the morphology of $\mathrm{Ti}_{3} \mathrm{C}_{2} \mathrm{~T}_{\mathrm{X}} \mathrm{MXene}$. As shown in Fig. $1 \mathrm{G}$ and $1 \mathrm{H}$, AFM image presented sheets with thickness of about $4 \mathrm{~nm}$, corresponding to the thickness of 3 layers.

\subsection{Electrocatalytic activity of $\mathrm{Ti}_{3} \mathrm{C}_{2} \mathrm{~T}_{\mathrm{x}} \mathrm{MXene}$ for phenolic compound oxidation}


The electrocatalytic activity for phenolic compound oxidation of $\mathrm{Ti}_{3} \mathrm{C}_{2} \mathrm{~T}_{\mathrm{x}} \mathrm{MXene}$ was confirmed by testing the electrocatalytic performances with different phenolic substrates, including 1-naphthol, 4-nitrophenol, and $\beta$-estradiol. As shown in Fig. 2 the modification of MXene significantly improved the oxidation currents for all the three phenolic compounds (DPV curves were seen in Supplementary Material, Fig. S2), indicating the favourable and comprehensive electrocatalytic activity of $\mathrm{Ti}_{3} \mathrm{C}_{2} \mathrm{~T}_{\mathrm{x}} \mathrm{MXene}$ for phenolic compound oxidation. Moreover, it's notable that the oxidation peaks presented distinct shifts to lower potential, revealing the electron transfer between $\mathrm{Ti}_{3} \mathrm{C}_{2} \mathrm{~T}_{\mathrm{x}} \mathrm{MXene}$ and phenolic compound.

To further profile the unique electrocatalytic activity of $\mathrm{Ti}_{3} \mathrm{C}_{2} \mathrm{~T}_{x} \mathrm{MXene,} \mathrm{several} \mathrm{nanomaterials} \mathrm{including}$ $\mathrm{MoS}_{2}$ nanosheets, $\mathrm{WS}_{2}$ nanosheets, $\mathrm{Fe}_{3} \mathrm{O}_{4}$ nanoparticles, $\mathrm{TiO}_{2}$ nanoparticles and bulk $\mathrm{Ti}_{3} \mathrm{AlC}_{2}$ were separately employed as constrast with 1-naphthol as substrate. It's notable that only $\mathrm{Ti}_{3} \mathrm{C}_{2} \mathrm{~T}_{\mathrm{x}} \mathrm{MXene}$ presented observable electrocatalytic activity for 1-naphthol oxidation, indicating that the unique electrocatalytic activity was the intrinsic property of $\mathrm{Ti}_{3} \mathrm{C}_{2} \mathrm{~T}_{x} \mathrm{MXene.}$

To quantitatively present the electrocatalytic activity of $\mathrm{Ti}_{3} \mathrm{C}_{2} \mathrm{~T}_{x} \mathrm{MXene}$ for 1-naphthol oxidation, DPV curves were measured with the addition of 1-naphthol at different concentrations. As shown in Fig. 3A, the peak current increased with the increasing concentration of 1-naphthol at low concentrations until reaching about $90 \mu \mathrm{A}$. According to Faraday's laws of electrolysis and Michaelis-Menten equation, the fitting curve was achieved with hyperbola function, where the Michaelis constant was calculated to be $0.22 \mathrm{mM}$, indicating that $\mathrm{Ti}_{3} \mathrm{C}_{2} \mathrm{~T}_{x}$ MXene possessed strong affinity to 1-naphthol[34]. Moreover, the catalytic activity of $\mathrm{Ti}_{3} \mathrm{C}_{2} \mathrm{~T}_{x}$ MXene for 1-naphthol oxidation in homogeneous phase solution was explored using hydrogen peroxide as oxidant. Fig. 3B displayed the real-time absorbance of the aqueous solutions at $387.5 \mathrm{~nm}$, including 1-naphthol (curve a), mixture of 1-naphthol and hydrogen peroxide (curve b), mixture of 1-naphthol, hydrogen peroxide and $\mathrm{Ti}_{3} \mathrm{C}_{2} \mathrm{~T}_{\mathrm{x}} \mathrm{MXene}$ (curve $\mathrm{c}$ ), respectively. It could be seen that the reaction ratio was significantly improved with the addition of $\mathrm{Ti}_{3} \mathrm{C}_{2} \mathrm{~T}_{\mathrm{x}} \mathrm{MXene}$, indicating that $\mathrm{Ti}_{3} \mathrm{C}_{2} \mathrm{~T}_{\mathrm{x}} \mathrm{MXene}$ could efficiently catalyze the 1-naphthol oxidation by hydrogen peroxide as well[35] .

To survey the quantitative relation between electrocatalytic activity and amount of $\mathrm{Ti}_{3} \mathrm{C}_{2} \mathrm{~T}_{\mathrm{x}} \mathrm{MXene}$, $\mathrm{Ti}_{3} \mathrm{C}_{2} \mathrm{~T}_{\mathrm{x}}$ MXene solutions at different concentrations were employed to modify GCE to test the electrocatalytic activities for 1-naphthol oxidation, respectively. As shown in Fig. 3C, the Faradic current increased linearly with the increasing concentration of $\mathrm{Ti}_{3} \mathrm{C}_{2} \mathrm{~T}_{\mathrm{x}} \mathrm{MXene}$ in low concentration range and reached a constant in high concentration range, indicating that the electrocatalytic activity was in direct proportion to the total modified area of $\mathrm{Ti}_{3} \mathrm{C}_{2} \mathrm{~T}_{\mathrm{x}}$ MXene flakes but not further improved by the stacking of the flakes.

\subsection{Mechanism study for the catalytic activity of $\mathrm{Ti}_{3} \mathrm{C}_{2} \mathrm{~T}_{\mathrm{x}}$ MXene}

The first principle calculations are performed to explain the catalytic activity of $\mathrm{Ti}_{3} \mathrm{C}_{2} \mathrm{~T}_{\mathrm{x}} \mathrm{MXene}$ for 1naphthol oxidation. The free energies of 1-naphthol adsorption were first calculated to determine whether 
1-naphthol could be adsorbed by $\mathrm{Ti}_{3} \mathrm{C}_{2} \mathrm{~T}_{\mathrm{x}} \mathrm{MXene}$. The optimized geometries of 1-naphthol on and $\mathrm{Ti}_{3} \mathrm{C}_{2} \mathrm{~T}_{x}$ MXene were given in Fig. S3 (see in Supplementary Material), which showed 1-naphthol molecule adsorbed on the plane surface of $\mathrm{Ti}_{3} \mathrm{C}_{2} \mathrm{~T}_{x} \mathrm{MXene}$ in a "lying-down" or "standing-up" manner, with adsorption energies of -1.18476 and $-0.75625 \mathrm{eV}$, respectively, indicating relative strong physical adsorption. As a result, the "lying-down" adsorption of 1-naphthol on $\mathrm{Ti}_{3} \mathrm{C}_{2} \mathrm{~T}_{\mathbf{x}} \mathrm{MXene}$ was critical in the electrocatalytic oxidation. To further investigate the origin of 1-naphthol adsorption, the differential charge density of 1-naphthol adsorbed on $\mathrm{Ti}_{3} \mathrm{C}_{2} \mathrm{~T}_{x}$ MXene was calculated. As shown in Fig. 3D, the changes in charge density caused by 1-naphthol adsorption mainly came from hydroxyl group and oxygen atoms. Moreover, changes in charge density were also found on the aromatic rings, indicating that the aromatic structure played a role in leading to the "lying-down" adsorption mode of 1-naphthol on $\mathrm{Ti}_{3} \mathrm{C}_{2} \mathrm{~T}_{\mathrm{x}}$ MXene.

\subsection{Optimization of experimental conditions}

To achieved optimal analytical performance of the biosensor, some critical experimental conditions were optimized, including the ratio of walker probe to support probe, and the cleaving time of Nt.BsmAl nicking endonuclease. As shown in Fig. 4, optimal ratio of walker probe to support probe and cleaving time was achieved to be 1:20 and $120 \mathrm{~min}$, respectively.

\subsection{Analytical performance of the proposed electrochemical biosensor}

To estimate analytical performance of the biosensor, the current responses toward BCR/ABL fusion gene at different concentrations were recorded under the optimal conditions through DPV measurements. As shown in Fig. $5 \mathrm{~A}$, the detection signal increased with the increasing concentration of target $\mathrm{BCR} / \mathrm{ABL}$ fusion gene. The corresponding calibration plots of the peak currents showed a strong linear relationship to the logarithm value of target $B C R / A B L$ fusion gene concentrations range from $0.2 \mathrm{fM}$ to $20 \mathrm{nM}$ with pearson correlation coefficient of 0.99836 (Fig. 5B). The linear regression equation was $I=1.00012 \times \mathrm{lg}$ $(c / p M)+11.23074$ ( $c$ and / stood for the concentration of target BCR/ABL fusion gene and corresponding peak current value, respectively). The limit of detection was obtained based on three times the average standard deviation corresponding to blank sample detection, which was calculated to be $0.05 \mathrm{fM}$. Comparisons of this biosensor with some reported works for BCR/ABL fusion gene detection are shown in Table S2, which highlighted the excellent sensitivity of this method in BCR/ABL fusion gene detection due to the cascading catalytic strategy and DNA walking machine for signal amplification.

Moreover, the specificity of the biosensor was evaluated by using 3 different DNA oligonucleotides as references, including a single-base-mismatched strand (B1), a two-base-mismatched strand (B2) and a noncomplementary strand (B3), all at concentrations of $20 \mathrm{nM}$. As depicted in Fig. 5C, the response signals of the single-base-mismatched strand and two-base-mismatched strand were much lower than the response signal of the target, revealing the good capacity of the biosensor to distinguish base- 
mismatch. The response signal of noncomplementary sequences were approximate to the blank solution, indicating that the biosensor presented good selectivity for DNA detection.

To evaluate the stability of the proposed biosensor, the modified electrodes were stored at $4^{\circ} \mathrm{C}$ before use. As presented in Fig. 5D, there were no obvious differences during the first 5 days of storage, and the current changes were less than $1.58 \%$. After 20 days of storage, the designed biosensor retained $89.40 \%$ of its initial current response, indicating that the proposed biosensor offers satisfactory stability for target $\mathrm{BCR} / \mathrm{ABL}$ detection.

\subsection{Detection of $B C R / A B L$ fusion gene in human serum samples}

To further validate the applicability of the biosensor to complex biological matrix in clinical application, different concentrations of target BCR/ABL fusion gene were added to 10-fold-diluted clinical serum samples and tested with the proposed biosensor. The detection results of BCR/ABL fusion gene in human serum samples are summarized in Table S3. Satisfactory recovery values were obtained ranging from 93.60-110.42\% with relative standard deviations (RSD) between $0.27 \%$ and $0.64 \%$. The results suggested that the proposed biosensor had the potential to be used for the determination of clinical samples.

\section{Conclusions}

In summary, this work reported the unique catalytic activity of $\mathrm{Ti}_{3} \mathrm{C}_{2} \mathrm{~T}_{x} \mathrm{MXene}$ nanozyme for phenols oxidation and the application to electrochemical biosensor for BCR/ABL fusion gene detection. The

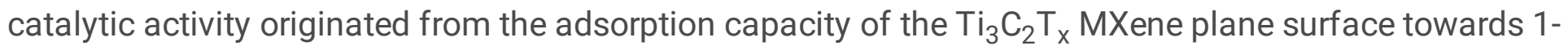
naphthol. Theoretic calculation revealed the mechanism that relatively strong interaction was existed between the surface oxygen atoms of $\mathrm{Ti}_{3} \mathrm{C}_{2} \mathrm{~T}_{\mathrm{x}}$ MXene and the phenolic hydroxyl groups. Moreover, the catalytic activity was strictly proportional to the cover area of the MXene fakes but independent to the number of stacking layers, which was quite different from traditional nanozymes and significant benefited the quality control of biosensor. Ultrasensitive detection was achieved with the proposed biosensor, which provided a promising analytical tool for the clinical diagnosis of CML.

\section{Declarations}

\section{Authors' contributions}

Rongjun Yu: Conceptualization, Methodology, Software, Investigation, Data Curation, Writing-Original Draft. Jian Xue: Software, Investigation, Project Administration. Yang Wang: Formal Analysis, Software. Jingfu Qiu: Supervision, Methodology, Data Curation, Administration. Xinyi Huang: Methodology, Software, Investigation, Project Administration. Anyi Chen: Writing-Review \& Editing. Jianjiang Xue: Funding Acquisition, Supervision, Administration, Data Curation. 


\section{Declaration of competing interest}

The authors declare no competing interests.

\section{Acknowledgments}

This research was supported by the National Natural Science Foundation of China (Grant Nos. 31071093, 31170129, and 31200064), China Postdoctoral Science Foundation (2020M683259) and the Science and Technology Planning Project of Yuzhong District of Chongqing city, China (No. 20140119).

\section{References}

1. Kimmel DW, LeBlanc G, Meschievitz M, E,Cliffel DE. Electrochemical Sensors Biosensors Anal Chem. 2012;84:685-707.

2. Maduraiveeran G, Sasidharan M, Ganesan V. Electrochemical sensor and biosensor platforms based on advanced nanomaterials for biological and biomedical applications. Biosens Bioelectron. 2018;103:113-29.

3. Rackus DG, Shamsi MH, Wheeler AR. Electrochemistry, biosensors and microfluidics: a convergence of fields. Chem Soc Rev. 2015;44:5320-40.

4. Wongkaew N, Simsek M, Griesche C, Baeumner AJ. Functional Nanomaterials and Nanostructures Enhancing Electrochemical Biosensors and Lab-on-a-Chip Performances: Recent Progress, Applications, and Future Perspective. Chem Rev. 2019;119:120-94.

5. Bocanegra-Rodriguez S, Molins-Legua C, Campins-Falco P, Giroud F, Gross AJ, Cosnier S. Monofunctional pyrenes at carbon nanotube electrodes for direct electron transfer $\mathrm{H} 2 \mathrm{O} 2$ reduction with HRP and HRP-bacterial nanocellulose. Biosens Bioelectron. 2021; 187.

6. Manesh KM, Santhosh P, Uthayakumar S, Gopalan A, I,Lee KP. One-pot construction of mediatorless bi-enzymatic glucose biosensor based on organic-inorganic hybrid. Biosens Bioelectron. 2010;25:1579-86.

7. Wang D, Chai Y, Yuan Y, Yuan R. Simple and Regulable DNA Dimer Nanodevice to Arrange Cascade Enzymes for Sensitive Electrochemical Biosensing. Anal Chem. 2020;92:14197-202.

8. Liu M, Chen G, Qin Y, Li J, Hu L, Gu W, Zhu C. Proton-Regulated Catalytic Activity of Nanozymes for Dual-Modal Bioassay of Urease Activity. Anal Chem.; 2021.

9. Jiang D, Ni D, Rosenkrans ZT, Huang P, Yan X. Cai W. Nanozyme: new horizons for responsive biomedical applications. Chem Soc Rev. 2019;48:3683-704.

10. Fan K, Wang H, Xi J, Liu Q, Meng X, Duan D, Gao L, Yan X. Optimization of Fe304 nanozyme activity via single amino acid modification mimicking an enzyme active site. Chem Commun. 2017;53:4247.

11. Liang M, Yan X, Nanozymes. From New Concepts, Mechanisms, and Standards to Applications. Acc Chem Res. 2019;52:2190-200. 
12. Huang K, Li Z, Lin J, Han G, Huang P. Two-dimensional transition metal carbides and nitrides (MXenes) for biomedical applications. Chem Soc Rev. 2018;47:5109-24.

13. Novoselov KS, Geim AK, Morozov SV, Jiang D, Zhang Y, Dubonos SV, Grigorieva IV, Firsov AA. Electric Field Effect in Atomically Thin Carbon Films. Science. 2004;306:666.

14. Jiang Y, Sun T, Xie X, Jiang W, Li J, Tian B, Su C Oxygen-Functionalized Ultrathin Ti3C2Tx MXene for Enhanced Electrocatalytic Hydrogen Evolution. ChemSusChem. 2019; 12: 1368-73.

15. Ma T, Cao J, Jaroniec M, Qiao S. Interacting Carbon Nitride and Titanium Carbide Nanosheets for High-Performance Oxygen Evolution. Angew Chem Int Ed. 2016;55:1138-42.

16. Yu M, Zhou S, Wang Z, Zhao J, Qiu J. Boosting Electrocatalytic Oxygen Evolution by Synergistically Coupling Layered Double Hydroxide with MXene. Nano Energy. 2017;44:181-90.

17. Zhao J, Zhang L, Xie X, Li X, Ma Y, Liu Q, Fang W, Shi X, Cui G, Sun X. Ti3C2Tx (T = F, OH) MXene nanosheets: conductive 2D catalysts for ambient electrohydrogenation of N2 to NH3. Journal of Materials Chemistry A. 2018;6:24031-5.

18. Handoko AD, Khoo KH, Tan TL, Jin H. Seh Z W. Establishing new scaling relations on twodimensional MXenes for CO2 electroreduction. J Mater Chem A. 2018;6:21885-90.

19. Jiang L, Duan J, Zhu J, Chen S, Antonietti M. Iron Clusters-Directed Synthesis of 2D/2D Fe-NC/MXene Superlattice-Like Heterostructure with Enhanced Oxygen Reduction Electrocatalysis. ACS Nano. 2020;14:2436-44.

20. Lang Z, Zhuang Z, Li S, Xia L, Zhao Y, Zhao Y, Han C, Zhou L. MXene Surface Terminations Enable Strong Metal-Support Interactions for Efficient Methanol Oxidation on Palladium. ACS Appl Mater Interfaces. 2020;12:2400-6.

21. Wang $\mathrm{H}$, Lee J. Recent advances in structural engineering of MXene electrocatalysts. J MaterChem A. 2020;8:10604-24.

22. Gao L, Fan K, Yan X. Iron Oxide Nanozyme: A Multifunctional Enzyme Mimetic for Biomedical Applications. Theranostics. 2017;7:3207-27.

23. Pratsinis A, Kelesidis GA, Zuercher S, Krumeich F, Bolisetty S, Mezzenga R, Leroux J-C. ,Sotiriou G A. Enzyme-Mimetic Antioxidant Luminescent Nanoparticles for Highly Sensitive Hydrogen Peroxide Biosensing. ACS Nano. 2017;11:12210-8.

24. Zhu Y, Zhang Z, Song X, Bu Y. A facile strategy for synthesis of porous Cu2O nanospheres and application as nanozymes in colorimetric biosensing. J Mater Chem B. 2021;9:3533-43.

25. Fan K, Xi J, Fan L, Wang P, Zhu C, Tang Y, Xu X, Liang M, Jiang B, Yan X. Gao L. In vivo guiding nitrogen-doped carbon nanozyme for tumor catalytic therapy. Nat Commun. 2018;9:1440.

26. Zhang P, Sun D, Cho A, Weon S, Lee S, Lee J, Han JW, Kim D-P. Choi W. Modified carbon nitride nanozyme as bifunctional glucose oxidase-peroxidase for metal-free bioinspired cascade photocatalysis. Nat Commun. 2019;10:940.

27. Chen T, Zou H, Wu X, Liu C, Situ B, Zheng L. Yang G. Nanozymatic Antioxidant System Based on MoS2 Nanosheets. ACS Appl Mater Interfaces. 2018;10:12453-62. 
28. Zhu J, Peng X, Nie W, Wang Y, Gao J, Wen W, Selvaraj JN, Zhang X, Wang S. Hollow copper sulfide nanocubes as multifunctional nanozymes for colorimetric detection of dopamine and electrochemical detection of glucose. Biosens Bioelectron. 2019;141:111450.

29. Xu Z, Liao L, Chai Y, Wang H, Yuan R. Ultrasensitive Electrochemiluminescence Biosensor for MicroRNA Detection by 3D DNA Walking Machine Based Target Conversion and DistanceControllable Signal Quenching and Enhancing. Anal Chem. 2017;89:8282-7.

30. Kresse G Furthmüller. J. Efficiency of ab-initio total energy calculations for metals and semiconductors using a plane-wave basis set. Comput Mater Sci. 1996;6:15-50.

31. Joubert D, Kresse G. From ultrasoft pseudopotentials to the projector augmented-wave method. Phys Rev B. 1999;59:1758-75.

32. Burke K, Ernzerhof M, Perdew JP Generalized Gradient Approximation Made Simple [Phys. Rev. Lett. 77, 3865 (1996)]. Phys Rev Lett 1997; 78: 1396-.

33. Grimme S, Antony J, Ehrlich S, Krieg H. A consistent and accurate ab initio parametrization of density functional dispersion correction (DFT-D) for the 94 elements H-Pu. J Chem Phys. 2010;132:154104.

34. Wang J, Huang R, Qi W, Su R, Binks BP, He Z. Construction of a bioinspired laccase-mimicking nanozyme for the degradation and detection of phenolic pollutants. Appl Catal B. 2019;254:452-62.

35. Jiang B, Duan D, Gao L, Zhou M, Fan K, Tang Y, Xi J, Bi Y, Tong Z, Gao GF, Xie N, Tang A, Nie G, Liang M. Yan X. Standardized assays for determining the catalytic activity and kinetics of peroxidase-like nanozymes. Nat Protoc. 2018;13:1506-20.

\section{Figures}



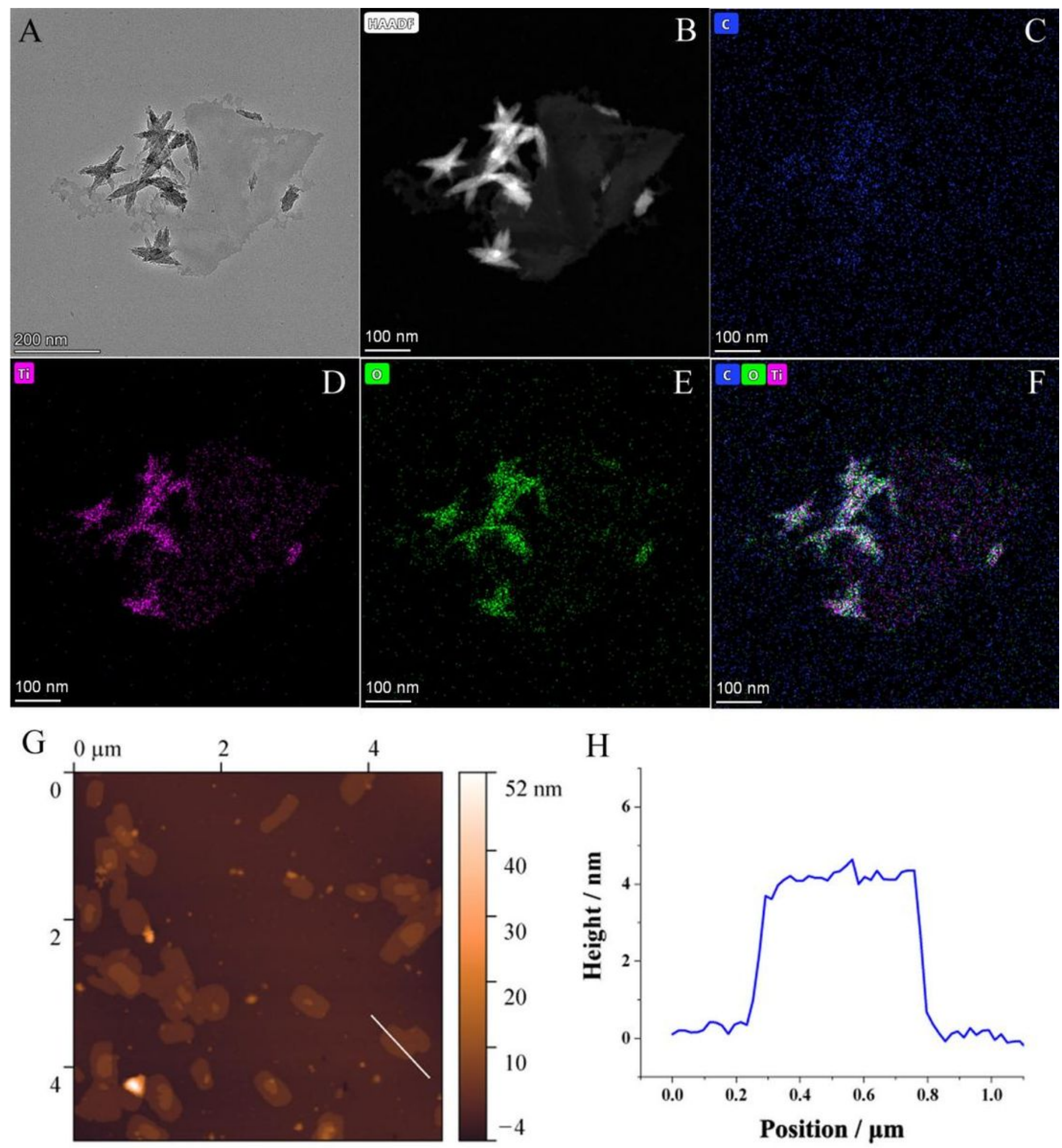

Figure 1

(A) TEM image, (B) HAADF-STEM image and (C-F) STEM-EDS elemental mappings of the Ti3C2TX MXene flakes. (G) AFM image of the Ti3C2Tx MXene flakes and $(\mathrm{H})$ the height profile along the white line. 

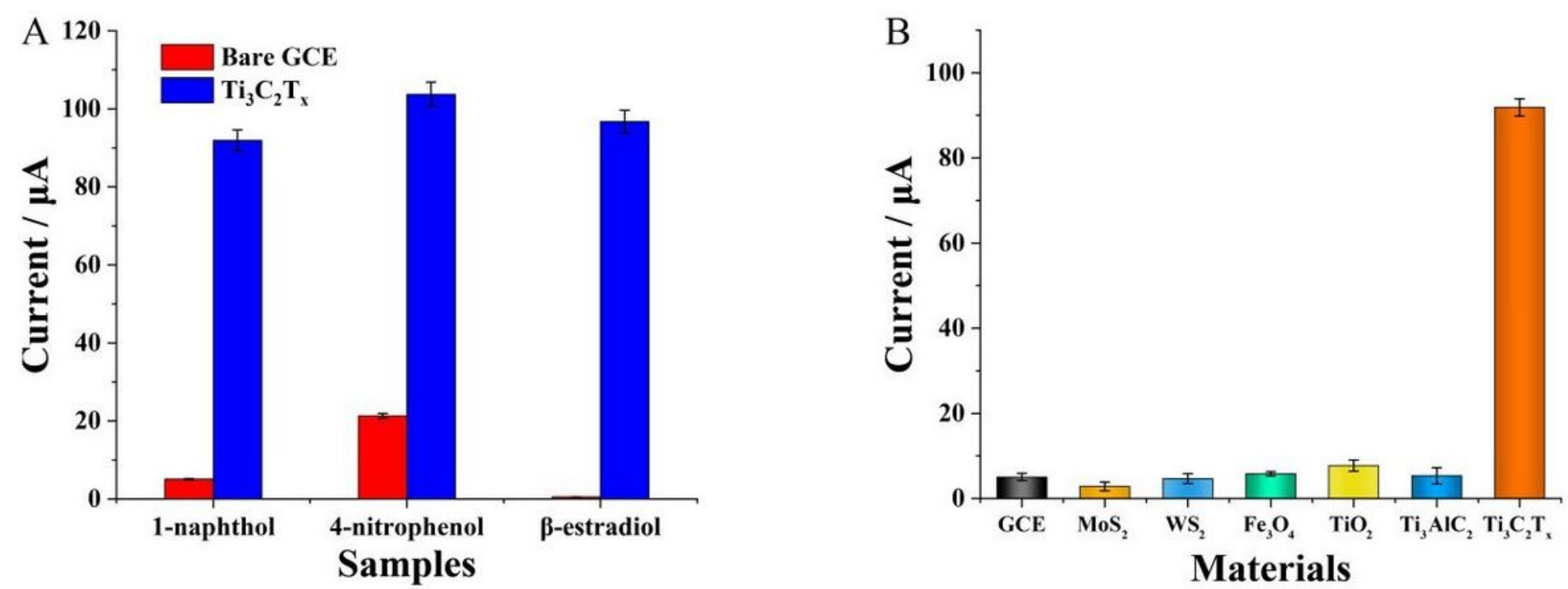

Figure 2

(A) Oxidation currents of different phenolic samples measured with bare GCE and Ti3C2Tx MXene modified electrode, respectively; (B) Oxidation current of 1-naphthol measured with different materials modified electrodes. 
A

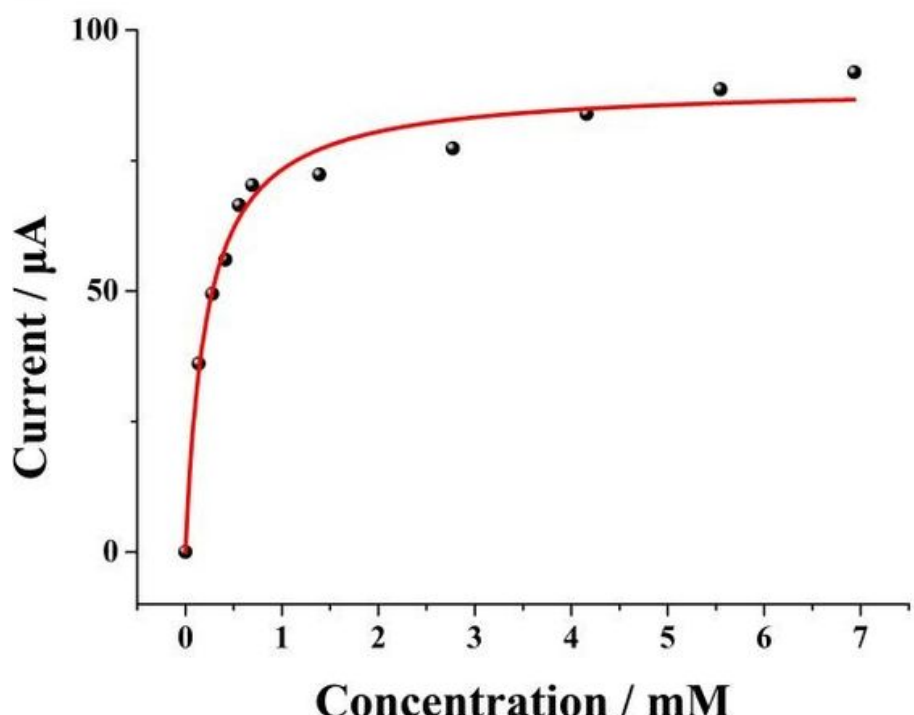

$\mathrm{C}$

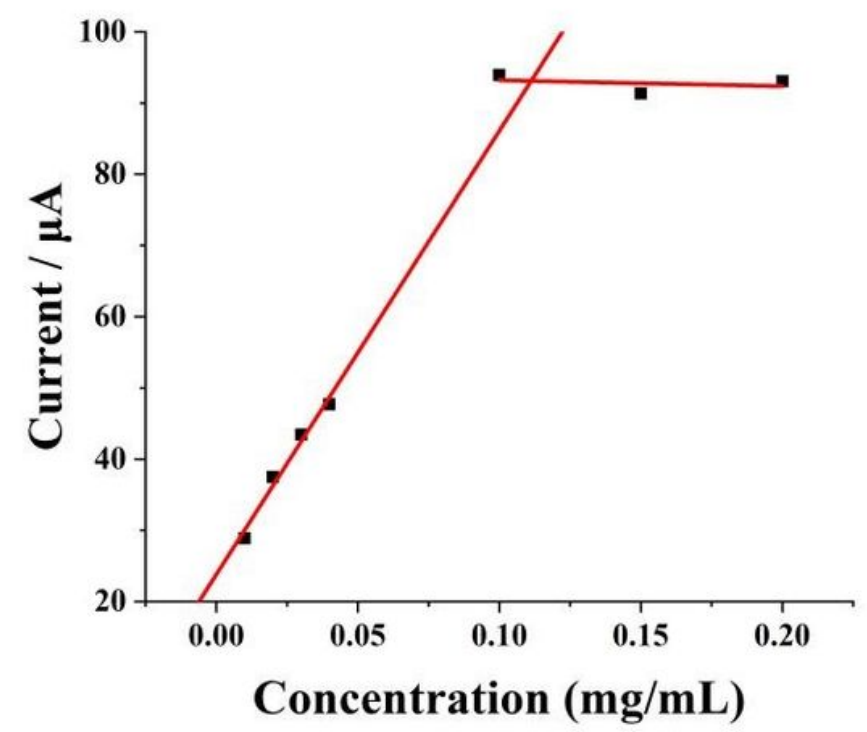

B

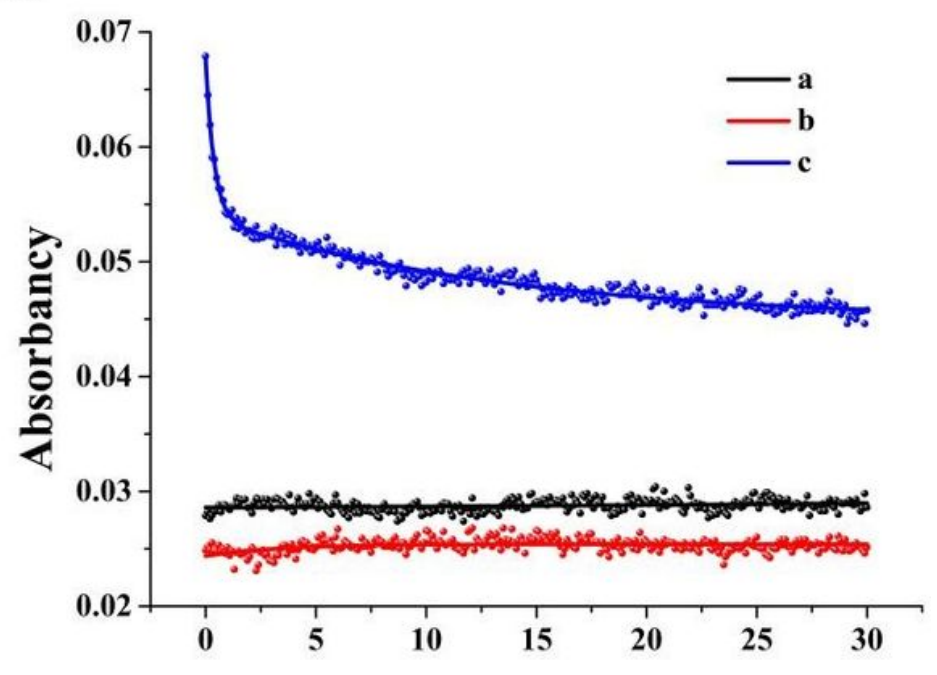

Time / $\min$

D

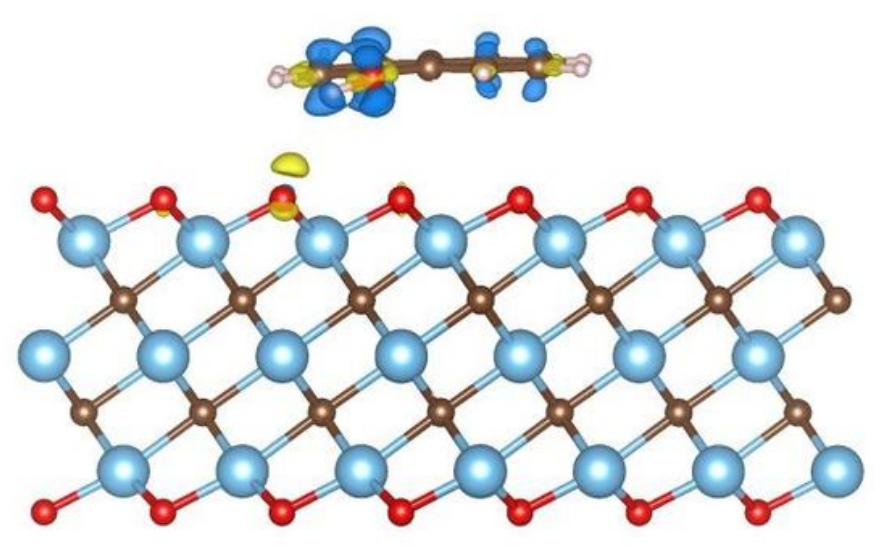

Figure 3

(A) Oxidation currents of 1-naphthol at different concentrations measured with Ti3C2Tx MXene modified electrode; (B) Absorbancy at $387.5 \mathrm{~nm}$ of 1-naphthol solution (curve a), mixture of 1-naphthol and hydrogen peroxide (curve b), mixture of 1-naphthol, hydrogen peroxide and Ti3C2Tx MXene (curve $c$ ), respectively. (C) The Faradic currents measured by electrode modified with Ti3C2Tx MXene at different concentrations in solution containing $7.0 \mathrm{mM}$ 1-naphthol. (D) Charge density difference of the "lyingdown" adsorption mode. Yellow and blue represent charge accumulation and depletion (isovalue: 0.005 au). 
A

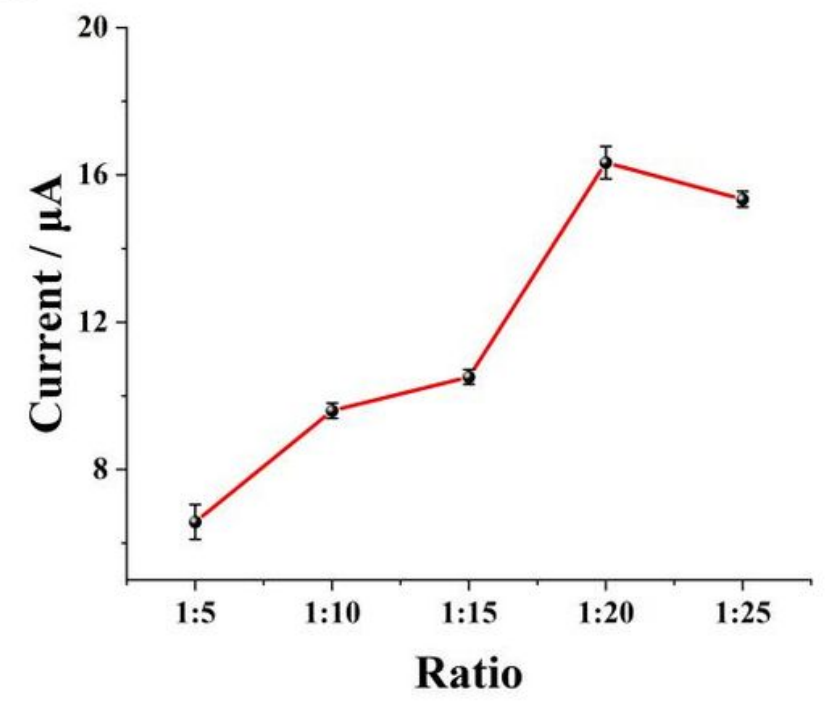

B

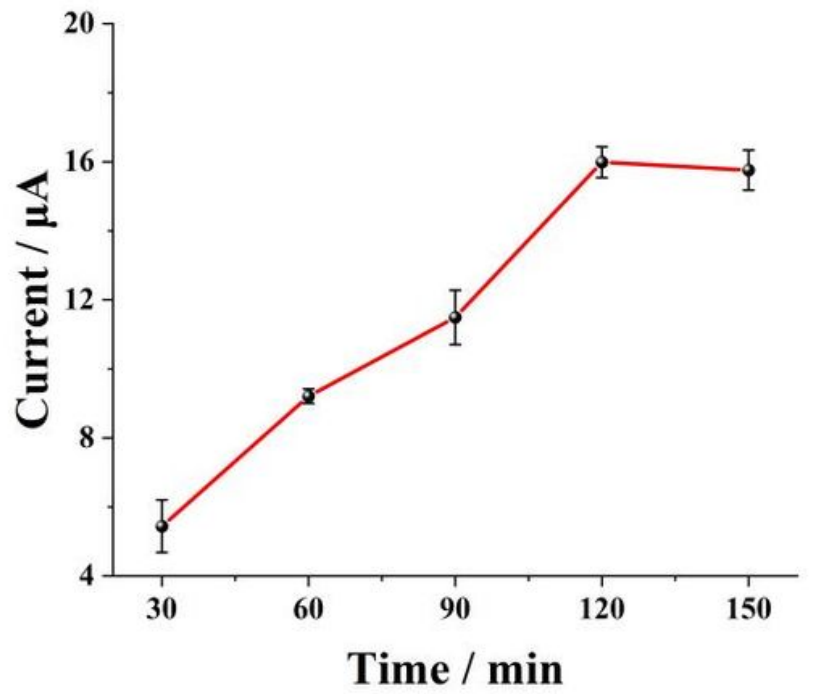

Figure 4

Optimization of experimental conditions: (A) ratio of walker to support DNA, (B) cleaving time of Nt.BsmAl nicking endonuclease. The error bars represent the standard deviation of three parallel measurements. 


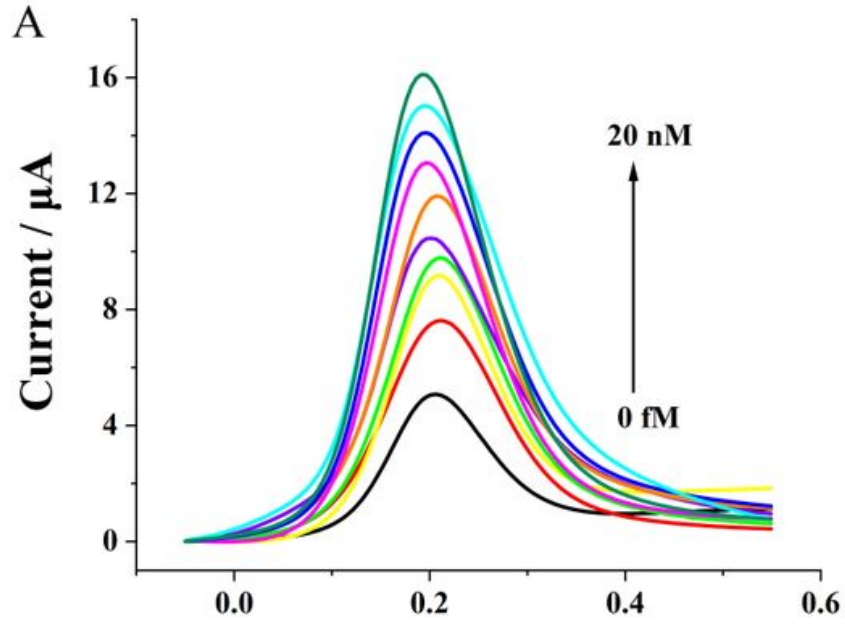

Potential / V

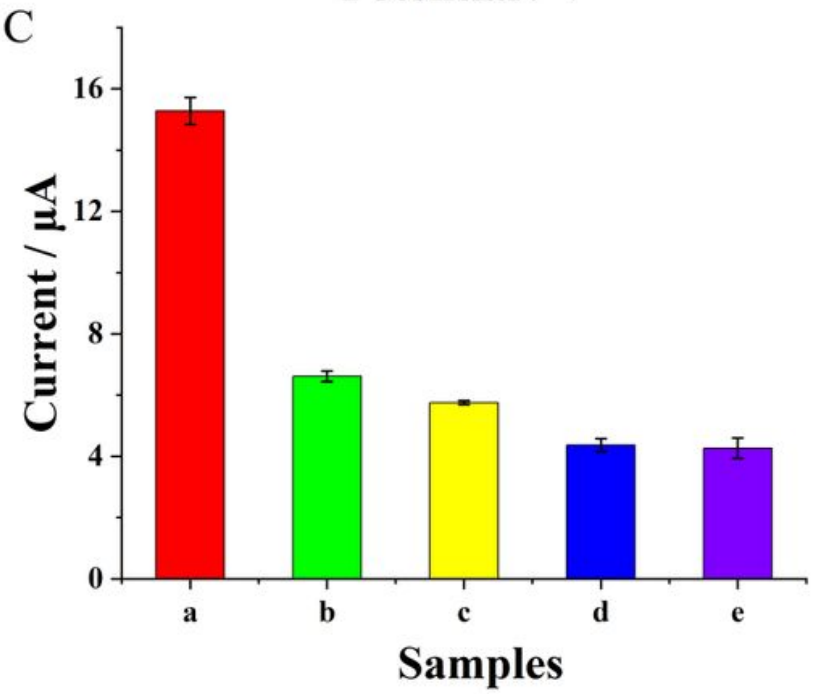

B

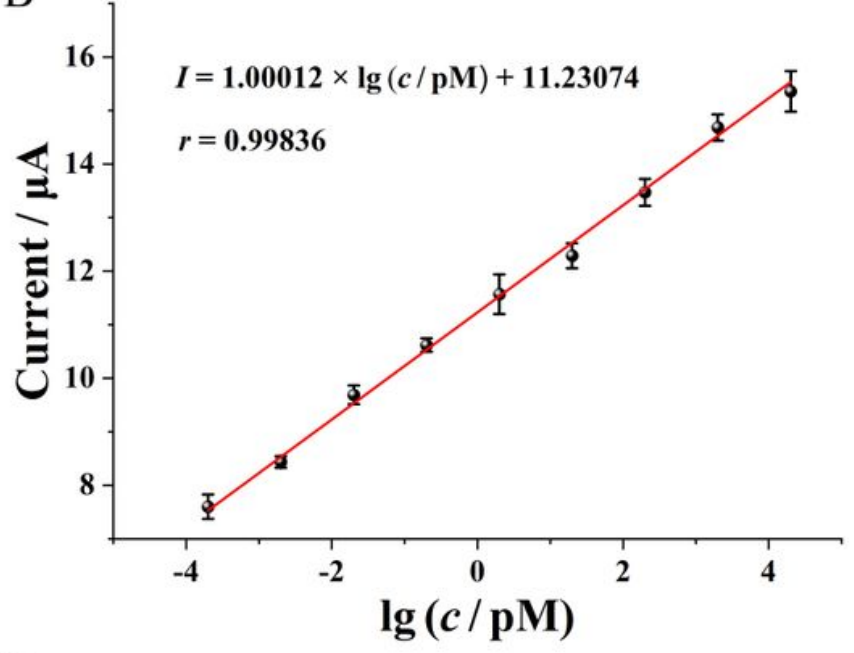

$\mathrm{D}$

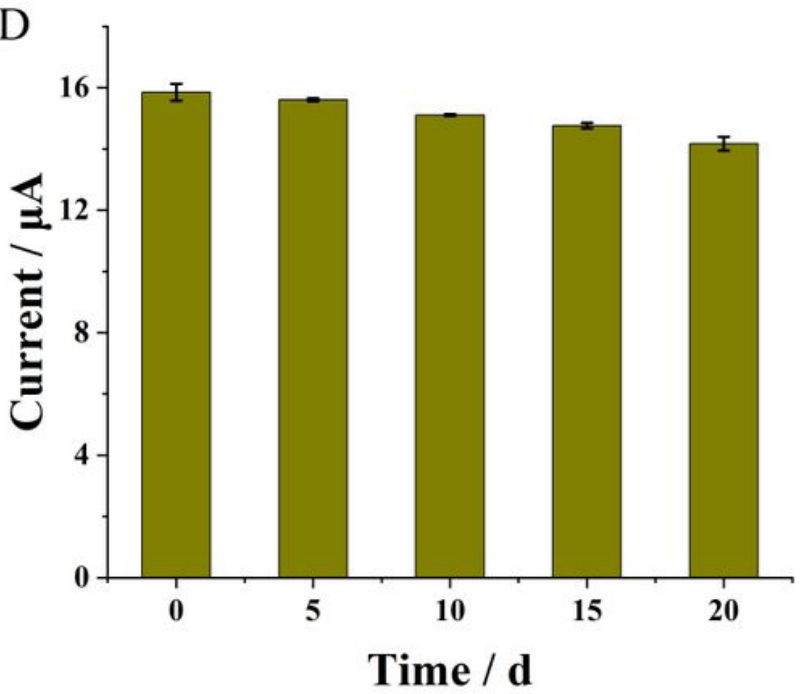

Figure 5

Evaluation of the sensitivity and specificity of the biosensor: (A) DPV curves response of the electrochemical biosensor upon the increase in target BCR/ABL fusion gene concentration (from bottom to top: $0 \mathrm{fM}, 0.2 \mathrm{fM}, 2 \mathrm{fM}, 20 \mathrm{fM}, 200 \mathrm{fM}, 2$ pM, 20 pM, 200 pM, and $2 \mathrm{nM}, 20 \mathrm{nM}$, separately) and (B) the corresponding linear relationship between DPV signal and logarithmic value of target BCR/ABL fusion gene concentrations. (C) DPV responses of the electrochemical biosensor to different oligonucleotides (20 nM): (a) BCR/ABL fusion gene (target), (b) single-base-mismatched strand (B1), (c) two-basemismatched strand (B2), (d) noncomplementary strand (B3), and (e) blank. (D) Stability of the proposed biosensor. The error bars represent the standard deviation of three parallel measurements.

\section{Supplementary Files}

This is a list of supplementary files associated with this preprint. Click to download.

- GraphicalAbstract.docx

- SupplementaryMaterial.docx 
- Scheme1.jpeg

Page 17/17 